\title{
Lattice Boltzmann model for photonic band gap materials
}

\author{
Zhifang Lin, Haiping Fang, Jianjun Xu, and Jian Zi \\ Research Center for Theoretical Physics and Department of Physics, Fudan University, Shanghai 200433, China
}

Xiangdong Zhang

Department of Physics, The Hong Kong University of Science and Technology, Hong Kong, China

(Received 7 November 2001; revised manuscript received 17 October 2002; published 7 February 2003)

An efficient technique for computing photonic band structure and defect modes is proposed based on the lattice Boltzmann model. Physically, it is a scheme based on the kinetics of the virtual microscopic process, rather than a solution of the macroscopic Maxwell equations. The method has significant advantages of being naturally suited for massively parallel machine, as well as speed and convenience, providing another methodology for photonic band gap materials and, also, for general electromagnetic scattering problems in open region when incorporated with the perfectly matched layer technique.

DOI: 10.1103/PhysRevE.67.025701

PACS number(s): 02.70.-c, 42.70.Qs, 41.20.Jb, 05.20.Dd

In the past decade, there has been much interest in artificially manufactured periodic dielectric media called photonic band gap (PBG) materials, since their unique optical properties enable one to manipulate and control the flow of photons [1-5].

Many methods have been proposed to study the photonic band structure and/or defect modes for PBG materials. Among others, the plane wave expansion method (PWEM) [6,7], multiple scattering theory (MST) [8], tight-binding formulation [9], transfer matrix method [10], and finite difference time domain (FDTD) scheme $[11,12]$ are well established. Most techniques are, physically, based on the solution of macroscopic Maxwell equations and, computationally, require time growing at a rate $N^{\eta}$, with $\eta \geqslant 2$ and $N$ being the number of expansion terms or discretization points in a cell. Here we set up a lattice Boltzmann (LB) model [13-15] for PBG materials, which is based on microscopic kinetics. The method has significant advantages of being naturally suited for massively parallel machines as well as requiring computation time scaling linearly with $N$ like FDTD. When incorporated with perfectly matched layer (PML) technique [16], it may also provide an alternative numerical tool for general electromagnetic scattering.

The model is defined on a two-dimensional (2D) square lattice of spacing $\Delta$. At time $t$, each lattice site $\mathbf{x}$ is associated with five quantities $f_{i}(\mathbf{x}, t), i=0,1,2,3,4$, which describe the distributions of virtual photons at site $\mathbf{x}$, time $t$, and moving with velocity $\Delta \mathbf{e}_{i} / \tau$. Here $\tau$ is the discrete time step on the lattice, $\mathbf{e}_{i}=\{\cos [2 \pi(i-1) / 4], \sin [2 \pi(i-1) / 4]\}$ for $i=1,2,3,4$, are four velocity directions along the links of the lattice, and $\mathbf{e}_{0}=0$.

The distribution functions evolve based on the LB Bhatnagar-Gross-Krook equation [13,17], which is rewritten into two procedures: relaxation,

$$
f_{i}^{+}(\mathbf{x}, t)=f_{i}(\mathbf{x}, t)+\Omega_{i}(\mathbf{x}, t),
$$

and streaming,

$$
f_{i}\left(\mathbf{x}+\Delta \mathbf{e}_{i}, t+\tau\right)=f_{i}^{+}(\mathbf{x}, t)
$$

where the collision term $\Omega_{i}(\mathbf{x}, t)$ takes the single relaxation time form, with the relaxation time $\xi$,

$$
\Omega_{i}(\mathbf{x}, t)=-\frac{1}{\xi}\left[f_{i}(\mathbf{x}, t)-f_{i}^{(0)}(\mathbf{x}, t)\right],
$$

and $f_{i}^{(0)}$ are local equilibrium distribution functions. The evolution rules (1) and (2) are particularly easy to implement on a massively parallel computer: the relaxation (1) can be computed simultaneously for each lattice site and then the streaming (2) is achieved with regular, local interprocessor communications.

Three macroscopic quantities are defined by

$$
\rho=\sum_{i=0}^{4} m_{i} f_{i}, \quad u_{\alpha}=\sum_{i=1}^{4} m_{i} f_{i} v e_{i \alpha},
$$

where $v=\Delta / \tau$, the Greek subscript $\alpha$ denotes the space directions in Cartesian coordinates, and $m_{i}$ are weights for different $f_{i}$. The physical symmetry suggests that for $i$ $=1,2,3,4, m_{i}=m$, which is set to unity for simplicity. The equilibrium distributions $f_{i}^{(0)}$ are chosen as

$$
\begin{gathered}
f_{i}^{(0)}=A \rho+\frac{B}{v} u_{\alpha} e_{i \alpha} \quad \text { for } i=1,2,3,4, \\
f_{0}^{(0)}=A_{0} \rho,
\end{gathered}
$$

where the coefficients $A, A_{0}, B$ are independent of $\rho, \mathbf{u}$ and can be determined by some macroscopic constraints. The first two are local conservation of $\rho$ and $\mathbf{u}$,

$$
\sum_{i=0}^{4} m_{i} f_{i}^{(0)}=\rho, \quad \sum_{i=1}^{4} f_{i}^{(0)} v e_{i \alpha}=u_{\alpha} .
$$

The macroscopic equations modeled by this virtual process can be worked out by performing a Chapman-Enskog expansion on the evolution equations (1)-(3) [13-15]. To second order, they are

$$
\partial_{t} \rho+\partial_{\alpha} u_{\alpha}=0,
$$




$$
\partial_{t} u_{\alpha}+2 A v^{2} \partial_{\alpha} \rho=0
$$

In deriving Eqs. (8) and (9), we have fixed $\xi=1 / 2$. Equations (8) and (9) are in fact the 2D Maxwell equations for the TM and TE cases if one sets

$$
\rho=\varepsilon E_{z}, \quad u_{x}=-H_{y}, \quad u_{y}=H_{x},
$$

and

$$
\rho=\mu H_{z}, \quad u_{x}=E_{y}, \quad u_{y}=-E_{x},
$$

respectively, and then requires

$$
2 A v^{2}=\frac{1}{\mu \varepsilon} .
$$

Here $\varepsilon$ and $\mu$ are, respectively, permittivity and permeability of the material. Equations (7) and (12) serve to determine the coefficients in Eqs. (5) and (6) [14]

$$
\begin{gathered}
A=\frac{1}{4 \mu_{r} \varepsilon_{r}}, \quad B=\frac{1}{2}, \\
A_{0}=\frac{\sqrt{\mu_{r} \varepsilon_{r}-1}}{2 \mu_{r} \varepsilon_{r}}, \quad m_{0}=2 \sqrt{\mu_{r} \varepsilon_{r}-1},
\end{gathered}
$$

where $\varepsilon_{r}=\varepsilon / \varepsilon_{0}$ and $\mu_{r}=\mu / \mu_{0}$ are relative permittivity and permeability of the material, respectively.

Based on the LB model, we have calculated the photonic band structures of square arrays of dielectric rods and perfectly conducting metal rods in air as well as a square array of air holes in dielectric. The lattice constant is $a$. The Bloch boundary conditions are

$$
\begin{aligned}
& f_{1}^{+}\left(\mathbf{x}-\mathbf{a}_{1}, t\right)=e^{-i \mathbf{k} \cdot \mathbf{a}_{1}} f_{1}^{+}(\mathbf{x}, t), \\
& f_{2}^{+}\left(\mathbf{x}-\mathbf{a}_{2}, t\right)=e^{-i \mathbf{k} \cdot \mathbf{a}_{2}} f_{2}^{+}(\mathbf{x}, t), \\
& f_{3}^{+}\left(\mathbf{x}+\mathbf{a}_{1}, t\right)=e^{i \mathbf{k} \cdot \mathbf{a}_{1}} f_{3}^{+}(\mathbf{x}, t), \\
& f_{4}^{+}\left(\mathbf{x}+\mathbf{a}_{2}, t\right)=e^{i \mathbf{k} \cdot \mathbf{a}_{2}} f_{4}^{+}(\mathbf{x}, t),
\end{aligned}
$$

with $\mathbf{a}_{1}=a(1,0), \mathbf{a}_{2}=a(0,1)$, and $\mathbf{k}$ the wave vector.

At interface between two different dielectrics, for the TE case, we replace the streaming (2) by

$$
\begin{gathered}
f_{1}\left(\mathbf{x}_{B}, t+\tau\right)=-\gamma f_{3}^{+}\left(\mathbf{x}_{B}, t\right)+(1-\gamma) f_{1}^{+}\left(\mathbf{x}_{A}, t\right), \\
f_{3}\left(\mathbf{x}_{A}, t+\tau\right)=\gamma f_{1}^{+}\left(\mathbf{x}_{A}, t\right)+(1+\gamma) f_{3}^{+}\left(\mathbf{x}_{B}, t\right),
\end{gathered}
$$

where $\gamma=\left(\varepsilon_{B}-\varepsilon_{A}\right) /\left(\varepsilon_{B}+\varepsilon_{A}\right)$, with $\varepsilon_{A}$ and $\varepsilon_{B}$ the permittivities at $\mathbf{x}_{A}$ and $\mathbf{x}_{B}=\mathbf{x}_{A}+\Delta \mathbf{e}_{1}$, respectively. Rule (14) resembles somewhat the method of images in electrostatics to consider the discontinuity effect of permittivity. It can be understood as some photons go through the interface while some are reflected by the interface. It applies to the TM case if all $\varepsilon$ in $\gamma$ are replaced by $\mu$. Similar rules are adopted for $f_{2}$ and $f_{4}$ when $\mathbf{x}$ and $\mathbf{x}+\Delta \mathbf{e}_{2}$ have different values of $\varepsilon$ and $\mu$.
When $\mathbf{x}+\Delta \mathbf{e}_{1}$ lies inside metal while $\mathbf{x}$ and all its other nearest neighbors are inside dielectric, we set

$$
f_{3}(\mathbf{x}, t)=-\sum_{i \neq 3} m_{i} f_{i}(\mathbf{x}, t)
$$

to force $E_{\tau}$, the tangential component of the $\mathbf{E}$ field, equal to zero for the TM case. If both $\mathbf{x}+\Delta \mathbf{e}_{1}$ and $\mathbf{x}+\Delta \mathbf{e}_{2}$ lie inside metal, we simply take

$$
f_{3}(\mathbf{x}, t)=f_{4}(\mathbf{x}, t)=-\frac{m_{0} f_{0}(\mathbf{x}, t)+f_{1}(\mathbf{x}, t)+f_{2}(\mathbf{x}, t)}{2} .
$$

For the TE case, in place of Eq. (15), we apply

$$
f_{3}(\mathbf{x}, t)=f_{1}(\mathbf{x}, t)
$$

to ensure $E_{\tau}=0$ at the surface of metal. While both $\mathbf{x}$ $+\Delta \mathbf{e}_{1}$ and $\mathbf{x}+\Delta \mathbf{e}_{2}$ are in metal,

$$
f_{3}(\mathbf{x}, t)=f_{2}(\mathbf{x}, t) \quad \text { and } \quad f_{4}(\mathbf{x}, t)=f_{1}(\mathbf{x}, t)
$$

yield a vanishing $E_{\tau}$ at metal surface for the TE mode. Similar rules apply to other interface cases.

The simulations begin by assigning a function called PEAKS in MATLAB function library to $E_{z}\left(H_{z}\right)$ to trigger every possible eigenmode for TM (TE) polarization. The temporal variation of fields can then be recorded based on the evolution rules (1)-(3) and (14)-(18). The spectral information is calculated using a discrete Fourier transform. The peaks of the spectral intensity correspond to the locations of eigenfrequencies. Figure 1(a) [1(b)] shows the band structures of a square array of dielectric rods (air holes) with radius $0.40 a$ in air (dielectric). The $\varepsilon_{r}$ of the dielectric is 11.7. Results for perfectly conducting metal rods are shown in Fig. 2. Excellent agreement with results obtained by the PWEM and MST validates the effectiveness of the LB model.

We next describe an LB model incorporated with the PML technique [16], which enables the simulation of openregion problems. To this end, we split $f_{0}$ into $f_{0 x}$ and $f_{0 y}$. The LB evolution equations (1)-(3) now read

$$
f_{i}\left(\mathbf{x}+\Delta \mathbf{e}_{i}, t+\tau\right)-f_{i}(\mathbf{x}, t)=\Omega_{i}(\mathbf{x}, t)+\Omega_{i}^{(a)}(\mathbf{x}, t),
$$

where $\Omega_{i}^{(a)}(\mathbf{x}, t)$ is introduced to consider the absorption assigned to the PML medium [16]. Four macroscopic quantities are defined

$$
\begin{gathered}
\rho_{x}=m_{0}^{\prime} f_{0 x}+f_{1}+f_{3}, \\
\rho_{y}=m_{0}^{\prime} f_{0 y}+f_{2}+f_{4}, \quad \mathbf{u}=\sum_{i=1}^{4} f_{i} v \mathbf{e}_{i} .
\end{gathered}
$$

The equilibrium distributions $f_{i}^{(0)}$ are given by

$$
f_{i}^{(0)}=A^{\prime}\left(\rho_{x}+\rho_{y}\right)+\frac{B^{\prime}}{v} u_{\alpha} e_{i \alpha} \quad \text { for } i=1,2,3,4
$$




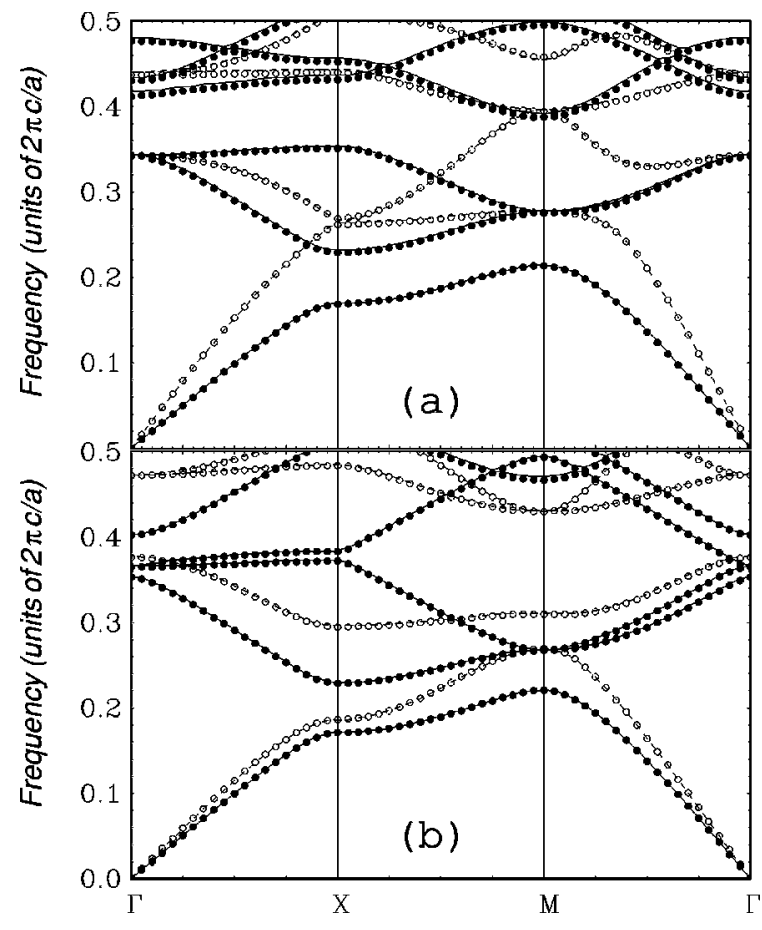

FIG. 1. The photonic band structure of a square array of dielectric rods in air (a) and air holes in dielectric (b), with both radii equal to $0.40 a$. The solid (dashed) lines are obtained by the PWEM for TM (TE) case. The solid and open circles are corresponding results by the LB model with $a=50 \Delta$ and 50000 time steps.

$$
f_{0 x}^{(0)}=A_{0}^{\prime} \rho_{x}-\frac{2 A^{\prime}}{m_{0}^{\prime}} \rho_{y}, \quad f_{0 y}^{(0)}=A_{0}^{\prime} \rho_{y}-\frac{2 A^{\prime}}{m_{0}^{\prime}} \rho_{x},
$$

with the coefficients

$$
\begin{gathered}
A^{\prime}=\frac{1}{4 \mu_{r} \varepsilon_{r}}, \quad B^{\prime}=\frac{1}{2}, \\
A_{0}^{\prime}=\frac{\sqrt{2 \mu_{r} \varepsilon_{r}-1}}{4 \mu_{r} \varepsilon_{r}}, \quad m_{0}^{\prime}=2 \sqrt{2 \mu_{r} \varepsilon_{r}-1} .
\end{gathered}
$$

By assuming $\Omega^{(a)} \sim \epsilon^{2}$, where $\epsilon$ is a small parameter of the order of the Knudsen number [18], four macroscopic equations result from a Chapman-Enskog expansion

$$
\begin{gathered}
\partial_{t} \rho_{x}+\partial_{x} u_{x}=\tilde{\sigma}_{x} \rho_{x}, \\
\partial_{t} \rho_{y}+\partial_{y} u_{y}=\tilde{\sigma}_{y} \rho_{y}, \\
\partial_{t} u_{\alpha}+2 A^{\prime} v^{2} \partial_{\alpha} \rho=\tilde{\sigma}_{\alpha} u_{\alpha},
\end{gathered}
$$

where use has been made of $\xi=1 / 2$ and

$$
\begin{array}{lll}
\Omega_{0 x}^{(a)}=\tau \tilde{\sigma}_{x} f_{0 x}^{(0)}, & \Omega_{i}^{(a)}=\tau \tilde{\sigma}_{x} f_{i}^{(0)} & \text { for } i=1,3, \\
\Omega_{0 y}^{(a)}=\tau \tilde{\sigma}_{y} f_{0 y}^{(0)}, & \Omega_{i}^{(a)}=\tau \tilde{\sigma}_{y} f_{i}^{(0)} & \text { for } i=2,4 .
\end{array}
$$

Equations (23) coincide with the Maxwell equations for TM and TE cases in the PML medium [16] if one sets

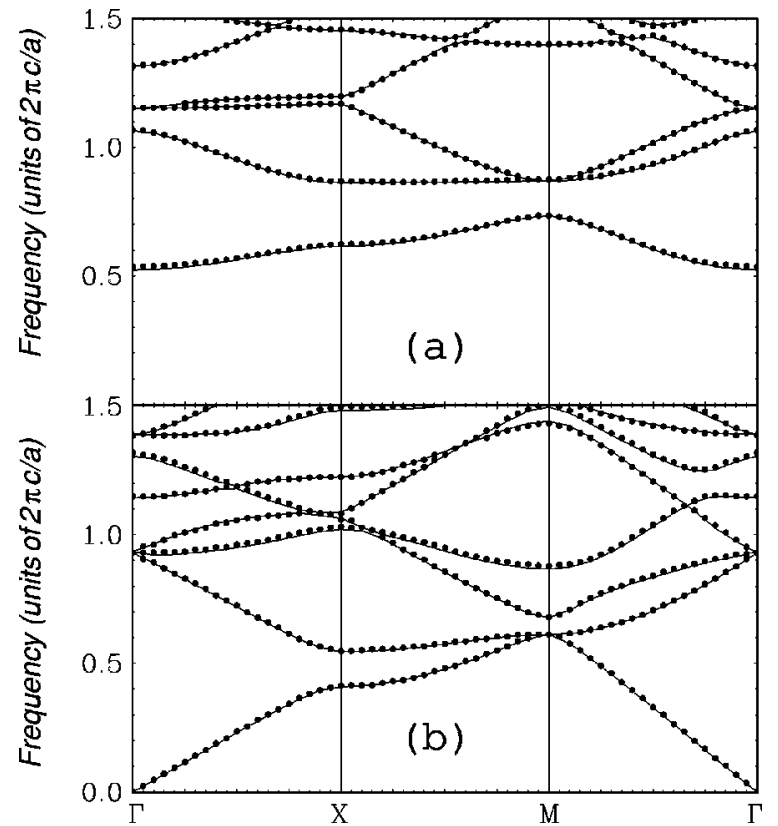

FIG. 2. The photonic band structure for TM (a) and TE (b) polarizations of a square array of perfectly conducting metal rods with radius $0.20 a$. The solid lines are obtained by MST while the circles by the LB model with 60000 time steps and $a=250 \Delta$ $(400 \Delta)$ for TM (TE) case.

$$
\rho_{x}=\varepsilon E_{z x}, \quad \rho_{y}=\varepsilon E_{z y}, \quad u_{x}=-H_{y}, \quad u_{y}=H_{x}
$$

and

$$
\rho_{x}=\mu H_{z x}, \quad \rho_{y}=\mu H_{z y}, \quad u_{x}=E_{y}, \quad u_{y}=-E_{x},
$$

respectively, and requires

$$
\tilde{\sigma}_{x}=-\frac{\sigma_{x}^{(e)}}{\varepsilon}=-\frac{\sigma_{x}^{(m)}}{\mu}, \quad \tilde{\sigma}_{y}=-\frac{\sigma_{y}^{(e)}}{\varepsilon}=-\frac{\sigma_{y}^{(m)}}{\mu} .
$$

Here $\left[\sigma_{x}^{(e)}, \sigma_{x}^{(m)}, \sigma_{y}^{(e)}, \sigma_{y}^{(m)}\right]$ are "pseudo" electric and magnetic conductivities assigned to absorb electromagnetic waves [16]. In the simulation, they must be set small enough to guarantee $\Omega^{(a)} \sim \epsilon^{2}$.

Based on the LB PML scheme, we have studied the defect modes in a square array of dielectric rods of refractive index 3.4 and radius $0.20 a$, which supports a large PBG for TM case [7]. Our simulation was performed on a computation domain $(\mathrm{CM})$ containing $7 \times 7$ unit cells with lattice constant $a=40 \Delta$. The CM is surrounded by a PML of thickness $80 \Delta$ with a smooth quadratic switching on of the absorption [16]. The maximum $\tilde{\sigma}_{x}$ and $\tilde{\sigma}_{y}$ are $0.25 / \tau$. The defect is introduced by changing the radius $r_{d}$ of the central rod in the CM. The simulations begin by assigning the value of the function PEAKS to $E_{z}$. The total number of time steps is 40000 . Figure 3 shows the frequency $\omega_{d}$ of the TM defect modes for various $r_{d}$. Figure 4 displays the electric field distributions of defect modes when $r_{d}=0.60 a$. The maximum of the elec- 


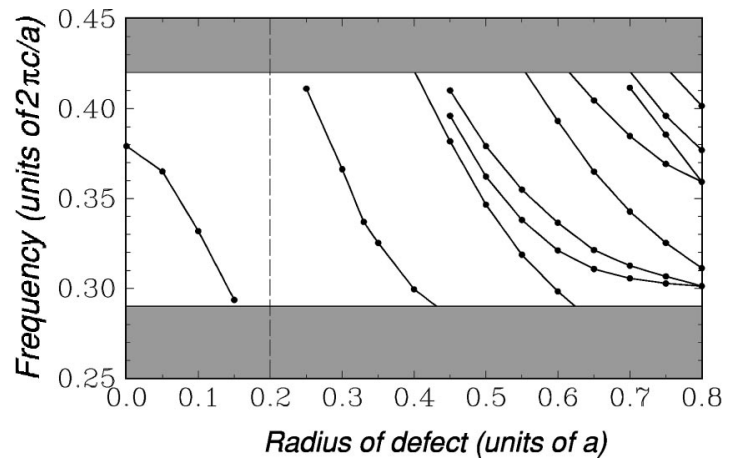

FIG. 3. Frequency $\omega_{d}$ of the TM defect modes in a square array of dielectric rods with radius $0.20 a$, obtained based on the LB PML model. The defect is introduced by changing the radius $r_{d}$ of a single rod. The shaded regions indicate the edges of the PBG.

tric field in each figure is normalized to unity in Figs. 4(a)4(d). Good agreement with results obtained by the PWEM [7] and FDTD [12] is found.

In conclusion, we have set up an LB model for PBG materials. The model provides an efficient scheme for handling not only periodic systems but also defects and disordered cases. An incorporation with the PML technique yields an LB PML scheme, which may be a promising numerical tool to simulate general electromagnetic scattering problems. In addition, the present scheme may open an avenue for the applications of the LB model. Several ways of further research were initiated: the first one is to extend the scheme to calculate transmission and reflection coefficients; the next lies in setting up an LB model for three-dimensional case;
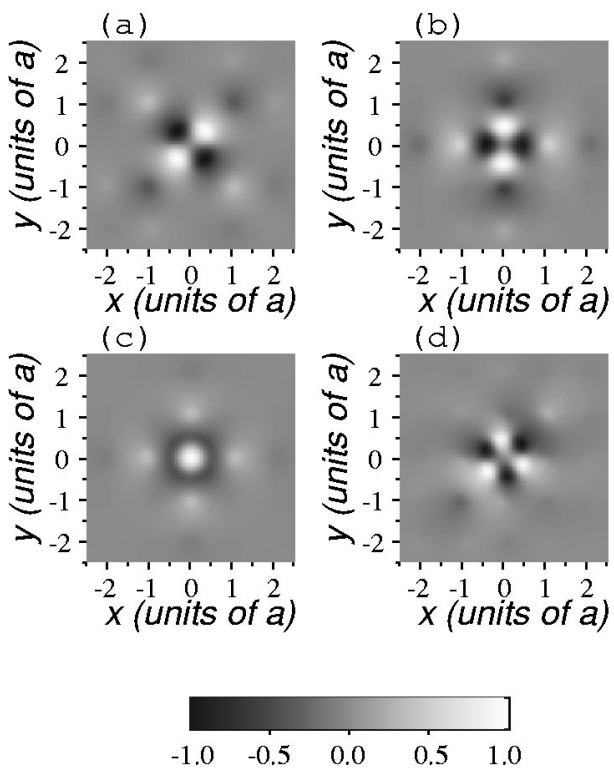

FIG. 4. Electric field distributions of the defect modes in Fig. 3 when $r_{d}=0.60 a$, obtained by the LB PML model. (a) Quadrupole, $\omega_{d}=0.298$; (b) quadrupole, $\omega_{d}=0.321$; (c) second-order monopole, $\omega_{d}=0.335$; and (d) hexapole, $\omega_{d}=0.392$. The frequency $\omega_{d}$ is in units of $(2 \pi c / a)$.

most appealing is to consider the nonlinear effect. As the LB method was initially designed for nonlinear fluid systems, we expect that it can be developed into an effective numerical scheme for nonlinear electromagnetic problems as well.

The work was supported by Climbing Project and NSFC through Grant Nos. 19704003 and 19834070.
[1] E. Yablonovitch, Phys. Rev. Lett. 58, 2059 (1987).

[2] S. John, Phys. Rev. Lett. 58, 2486 (1987).

[3] Photonic Band Gaps and Localization, edited by C. M. Soukoulis (Plenum, New York, 1993); J. Opt. Soc. Am. B 10, 283 (1993); J. Mod. Opt. 41, 171 (1994); Photonic Band Gap Materials, edited by C. M. Soukoulis (Kluwer, Dordrecht, 1996).

[4] J. Joannopoulos, R.D. Meade, and J. Winn, Photonic Crystals (Princeton University Press, Princeton, 1995).

[5] A. Mekis et al., Phys. Rev. Lett. 77, 3787 (1996).

[6] K.M. Ho et al., Phys. Rev. Lett. 65, 3152 (1990); K.M. Leung and Y.F. Liu, ibid. 65, 2646 (1990); Z. Zhang and S. Satpathy, ibid. 65, 2650 (1990); M. Plihal et al., Opt. Commun. 80, 199 (1991); R.D. Meade et al., Phys. Rev. B 48, 8434 (1993).

[7] P.R. Villeneuve et al., Phys. Rev. B 54, 7837 (1996).

[8] X. Wang et al., Phys. Rev. B 47, 4161 (1993); K.M. Leung and Y. Qiu, ibid. 48, 7767 (1993); W.Y. Zhang et al., Phys. Rev. Lett. 84, 2853 (2000).

[9] E. Lidorikis et al., Phys. Rev. Lett. 81, 1405 (1998).

[10] J.B. Pendry and A. Mackinnon, Phys. Rev. Lett. 69, 2772 (1992); M.M. Sigalas et al., Phys. Rev. B 48, 14121 (1993); ibid. 49, 11080 (1994).

[11] C.T. Chan et al., Phys. Rev. B 51, 16635 (1995); V. Kuzmiak and A.A. Maradudin, ibid. 57, 15242 (1998); A.J. Ward and J.B. Pendry, ibid. 58, 7252 (1998); Comput. Phys. Commun. 128, 590 (2000).

[12] M. Qiu and S. He, Phys. Rev. B 61, 12871 (2000).

[13] Y.H. Qian, D. d'Humiéres, and P. Lallemand, Europhys. Lett. 17, 479 (1992); H. Chen, S. Chen, and W.H. Matthaeus, Phys. Rev. A 45, R5339 (1992).

[14] B. Chopard and M. Droz, Cellular Automata Modeling of Physical Systems (Cambridge University Press, Cambridge, 1998).

[15] S.Y. Chen and G.D. Doolen, Annu. Rev. Fluid Mech. 30, 329 (1998).

[16] J.P. Berenger, J. Comput. Phys. 114, 185 (1994).

[17] P.L. Bhatnagar, E.P. Gross, and M. Krook, Phys. Rev. 94, 511 (1954).

[18] S.P. Dawson, S. Chen, and G.D. Doolen, J. Chem. Phys. 98, 1514 (1993). 